\title{
Management of incidental unruptured intracranial aneurysms
}

\author{
Shelley Renowden, Richard Nelson
}

Department of Neurosurgery, Southmead Hospital, Bristol, UK

\section{Correspondence to}

Shelley Renowden; Department of Neurosurgery, Southmead Hospital, Bristol WC1H 9JR, UK; shelley.renowden@nbt.nhs.uk

Accepted 3 June 2020

\section{Check for updates}

(C) Author(s) (or their employer(s)) 2020. No commercial re-use. See rights and permissions. Published by BMJ.

To cite: Renowden $S$, Nelson R. Pract Neurol 2020;20:347-355.

\begin{abstract}
The widespread use of MR has led to the increasingly frequent diagnosis of unruptured incidental intracranial aneurysms. Most are small ( $<7 \mathrm{~mm}$ diameter) and will never rupture. Yet, their recognition causes much anxiety, and their optimal management remains controversial. This review addresses the difficulties in managing incidental unruptured saccular intracranial aneurysms. Note that our conclusions and recommendations do not apply to symptomatic unruptured aneurysms or to fusiform, dissecting, mycotic, traumatic and paediatric aneurysms, each of which has a different natural history.
\end{abstract}

\section{EPIDEMIOLOGY}

Incidental unruptured intracranial aneurysms (UIAs) are acquired vascular lesions that develop most frequently at the branching of the basal cerebral arteries, in patients usually between the fourth and sixth decades of life. They occur in 3\% $95 \%$ CI $1.9 \%$ to $5.2 \%$ ) of the adult population (mean age 50 years), ${ }^{1}$ being twice as common in women than men, and are rare before the age of 20 years. Between 20\% and $30 \%$ of affected patients have multiple UIAs. Their increasing rate of diagnosis contrasts with the worldwide fall in the incidence of aneurysmal subarachnoid haemorrhage-by $40 \%$ between 1980 and 2010 (from 10.2 to 6.1 per 100000 ) - in parallel with a global decrease in mean blood pressure and smoking prevalence. ${ }^{2}$

\section{PATHOPHYSIOLOGY}

The factors involved in forming UIAs include a genetic predisposition, local haemodynamic stress, inflammatory changes and endothelial dysfunction promoted by smoking and hypertension, which both generate oxidative stress. ${ }^{3}$ In the cellular pathophysiology of aneurysm formation, endothelial dysfunction leads to an inflammatory response and phenotypic demodulation of vascular smooth muscle cells. There is disruption of the internal elastic lamina, dysregulation of collagen synthesis, vascular smooth muscle cell apoptosis and thinning of the media. Macrophages express and release matrix metalloproteases that cleave the extracellular matrix and collagen of the arterial wall.

Aneurysm stability then depends on the balance between repair (due to eutrophic collagen turnover, vascular smooth muscle cell proliferation and regeneration of the extracellular matrix) and wall destruction (due to dystrophic collagen turnover and degradation of the extracellular matrix).

\section{RISK OF RUPTURE}

The rationale for treating an UIA is to prevent the potentially devastating effects of aneurysmal subarachnoid haemorrhage. The decision to treat depends on its perceived risk of rupture. Assuming an aneurysmal prevalence of 3\% (3000/100 000 population) and an annual incidence of aneurysmal subarachnoid haemorrhage of 6-10/100 000 population, only one UIA will rupture each year for every 300-500 persons with an aneurysm. Thus, a critically important part of clinical decision-making is to identify the subgroup of patients at most risk of UIA rupture.

Our ability to identify that subgroup and to estimate accurately an individual patient's rupture risk remains imperfect, despite decades of epidemiological research. The most robust information currently available Population, Hypertension, Age, Sex, Earlier subrachnoid haemorrhage and Site (PHASES) ${ }^{5}$ is from a pooled analysis of over 10272 UIAs in 8882 patients from six prospective cohort studies, including the International Study of Unruptured Intracranial Aneurysms. ${ }^{6}$ Using the PHASES score (table 1), the mean 1-year risk of rupture of all UIAs is $1.4 \%$ (95\% CI $1.1 \%$ to $1.6 \%$ ) and five-year risk is $3.4 \%$ (95\% CI $2.9 \%$ to $4.0 \%$ ).

A recent prospective population-based study of a cohort of 841 patients with both unruptured and ruptured aneurysms showed that a PHASES score of $\geq 3 / 4$ 


\begin{tabular}{|c|c|}
\hline North American, European (not Finnish) & 0 \\
\hline Japanese & 3 \\
\hline Finnish & 5 \\
\hline Hypertension, yes & 1 \\
\hline $70+$ years old & 1 \\
\hline$<7 \mathrm{~mm}$ & 0 \\
\hline 7.0-9.9 mm & 3 \\
\hline $10.0-19.9 \mathrm{~mm}$ & 6 \\
\hline $20 \mathrm{~mm}+$ & 10 \\
\hline Previous aneurysmal SAH (another aneurysm) & 1 \\
\hline Site-internal carotid artery & 0 \\
\hline Middle cerebral artery & 2 \\
\hline Anterior cerebral artery/PCom artery/posterior circulation & 4 \\
\hline Risk score & $\begin{array}{l}5 \text {-year risk } \\
\text { of rupture } \%\end{array}$ \\
\hline 2 & 0.4 \\
\hline 3 & 0.7 \\
\hline 4 & 0.9 \\
\hline 5 & 1.3 \\
\hline 6 & 1.7 \\
\hline 7 & 2.5 \\
\hline 8 & 3.2 \\
\hline 9 & 4.3 \\
\hline 10 & 5.3 \\
\hline 11 & 7.2 \\
\hline 12 & 17.8 \\
\hline
\end{tabular}

PCom, posterior communicating; SAH, subarachmoid haemorrhage

could distinguish between groups of UIA patients at low and high rupture risk. ${ }^{7}$

PHASES should be used only as a guide because several other risk factors discussed below are not included.

Aneurysmal size (maximum luminal diameter in $\mathrm{mm}$ ) is one of the most important factors determining rupture risk. PHASES established that this risk rises above a threshold diameter of $7 \mathrm{~mm}$. Others confirmed that $85 \%$ of aneurysms with diameter below $7 \mathrm{~mm}$ remained stable and did not grow during 10 years of follow-up ${ }^{8}$; aneurysms measuring $\geq 5 \mathrm{~mm}$ have an increased risk of haemorrhage. ${ }^{9}$ A diameter of $5-7 \mathrm{~mm}$ is generally considered the threshold for treatment but the decision also rests on other factors such as age, aneurysm location and morphology, family history and comorbidities.

Smoking is a well-established risk factor for aneurysm formation, growth and rupture of UIA, with a linear, dose-dependent association. However, smoking and other potentially important risk factors-heavy alcohol consumption, aneurysm morphology, aneurysm growth, inflammation, family history, female sex and aneurysm multiplicity-could not be included in the PHASES analysis due to incomplete or heterogeneously defined data.

\section{ANEURYSM GROWTH}

Aneurysmal growth and change in morphology are regarded as markers of instability leading to an increased propensity to rupture. The risk of a growing aneurysm rupturing is 5 to 55 times that of a stable aneurysm. ${ }^{810-12}$

Following their initial detection, $10-12 \%$ of UIAs grow within 3 years, rising to $45 \%$ after 19 years. ${ }^{11}{ }^{13-15}$ Initial aneurysm size is an important factor for subsequent growth. Aneurysm enlargement is variably defined but an increase of at least $1 \mathrm{~mm}$ in any direction is generally accepted to be significant. Some authors consider the enlargement important if a $\leq 5 \mathrm{~mm}$ aneurysm increases by $1 \mathrm{~mm}$ or a $>5 \mathrm{~mm}$ aneurysm increases by $2 \mathrm{~mm} .{ }^{11}$

Aneurysm growth is non-linear, often with long periods of stability and short periods of aneurysm growth. The duration of increased risk of rupture after enlargement is unknown and the absolute risk may still be small.

The major risk factors for aneurysmal growth are smoking and aneurysm size $>5 \mathrm{~mm}$. Hypertension, aneurysm location on the posterior circulation, multilobulation, age $>50$ years, female sex, dome/neck diameter ratio $>1$ and excessive alcohol consumption also play a role. ${ }^{11} 16$

Based on data from 1507 conservatively managed patients with 1909 UIAs, from 10 centres in five countries with a follow-up of 5782 patient years, ${ }^{10}$ the Earlier subarachnoid haemorrhage, Location, Age, Population, Size and Shape (ELAPSS) score (table 2) uses reported risk factors to predict aneurysmal growth at 3 and 5 years. This score and the PHASES score for rupture share many risk factors and are highly correlated. Higher PHASES scores are associated with an increased risk of aneurysm growth. ${ }^{17}$

High-quality prospective cohort studies consistently report that aneurysm morphology and irregularity

\begin{tabular}{|c|c|c|}
\hline $\begin{array}{l}\text { Earlier aneurysmal } \\
\text { subarachnoid haemorrhage }\end{array}$ & Yes & 1 \\
\hline \multirow[t]{2}{*}{ Location } & $\begin{array}{l}\text { Middle cerebral } \\
\text { artery }\end{array}$ & 3 \\
\hline & $\begin{array}{l}\text { PCom artery/ } \\
\text { posterior circulation }\end{array}$ & 5 \\
\hline$>60$ years old & & 1 \\
\hline Japanese & & 1 \\
\hline Finnish & & 7 \\
\hline \multirow[t]{5}{*}{ Size } & $1.0-2.9 \mathrm{~mm}$ & 0 \\
\hline & $3.0-4.9 \mathrm{~mm}$ & 4 \\
\hline & $5.0-6.9 \mathrm{~mm}$ & 10 \\
\hline & $7.9-9.0 \mathrm{~mm}$ & 13 \\
\hline & $>10 \mathrm{~mm}$ & 22 \\
\hline \multirow[t]{2}{*}{ Irregular } & Yes & 4 \\
\hline & $\begin{array}{l}\text { 3-year risk of } \\
\text { growth \% }\end{array}$ & $\begin{array}{l}\text { 5-year risk of } \\
\text { growth\% }\end{array}$ \\
\hline$<5$ & 5 & 8 \\
\hline $5-9$ & 8 & 13 \\
\hline $15-19$ & 18 & 28 \\
\hline $20-24$ & 26 & 40 \\
\hline$>25$ & 43 & 61 \\
\hline
\end{tabular}


(daughter aneurysms and blebs) are risk factors for rupture, but the magnitude of this risk is not known. Casecontrol studies have identified two factors associated with rupture: size ratio $>3$ (maximal aneurysm diameter/parent artery diameter) and aspect ratio (aneurysm height/ neck width perpendicular to height), but these await validation in larger prospective cohorts. The aspect ratio for ruptured aneurysms averages 2.4 , while the ratio for UIAs is typically $<1.6 .^{18} 19$

\section{MANAGEMENT}

We must not underestimate the psychological impact on individual patients of the discovery of an asymptomatic UIA. A Google search on 'unruptured brain aneurysm' in the UK leads first to the National Health Service (NHS) website (https://www.nhs.uk/condi tions/brain-aneurysm/symptoms/) where a single web page, while factually correct, juxtaposes symptoms of an unruptured brain aneurysm with (a) ruptured brain aneurysms and (b) medical emergency. The 'ticking time bomb' analogy is never far away. There is a strong case for all patients to receive timely counselling, based on a multidisciplinary neurovascular team review of their risk factors and management options.

For most patients, it is easier to quantify the risks of treatment than to predict the natural history of their UIA.
A randomised controlled trial comparing active and conservative management was attempted some years ago (Trial of Endovascular Aneurysm Management) ${ }^{20}$ but was halted because of slow recruitment. As such, management recommendations have to be based on the metaanalysis of cohort studies, natural history data and decision-making scores and algorithms.

\section{ENDOVASCULAR TREATMENT}

Standard endovascular coiling (figure 1), with balloon or stent assistance, continues to be the mainstay of treatment for most UIAs. The main risks associated with endovascular therapy are procedural thromboembolic events (around 7\%) and aneurysmal rupture $(2-3 \%){ }^{21}$ Flow diversion stents (figure 2) generally have more complications, with procedurerelated morbidity of 5\% (95\% CI $4 \%$ to $7 \%$ ) and mortality of $4 \%(95 \%$ CI $3 \%$ to $6 \%))^{22}$ More recently introduced intrasaccular devices such as the woven endobridge device (figure 3) have similar morbidity rates of up to $5 \% .^{23}$

In a meta-analysis of 71 studies comprising 5044 patients with 5771 UIAs that included simple and assisted coiling, ${ }^{24}$ endovascular therapy was associated with unfavourable outcomes including death in $4.8 \%$ of cases (95\% CI 3.9\% to 6.0\%). Thromboembolic a

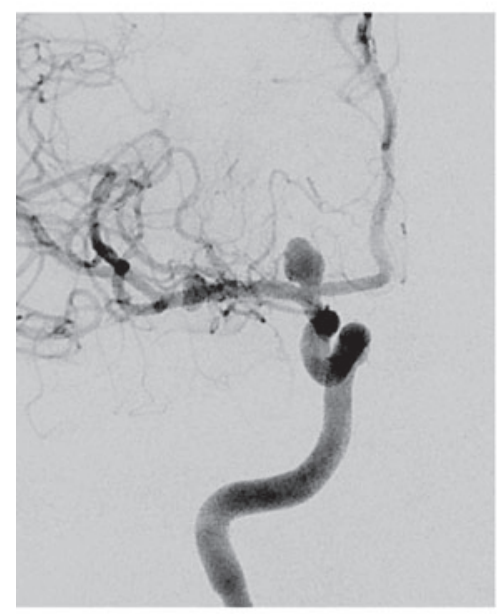

\section{b}

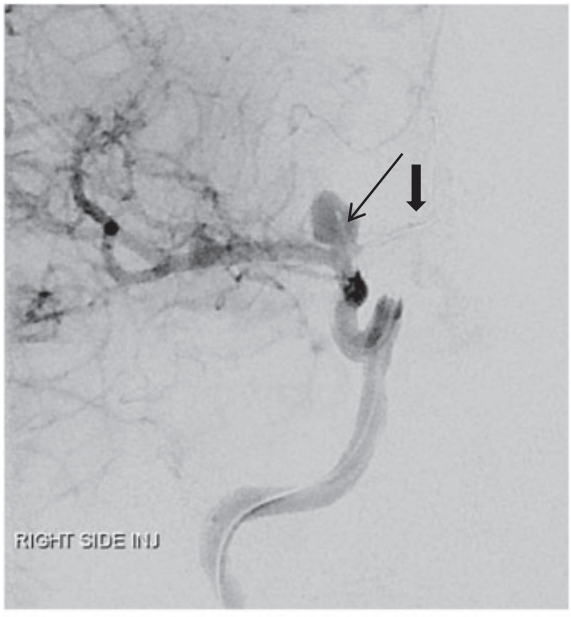

d
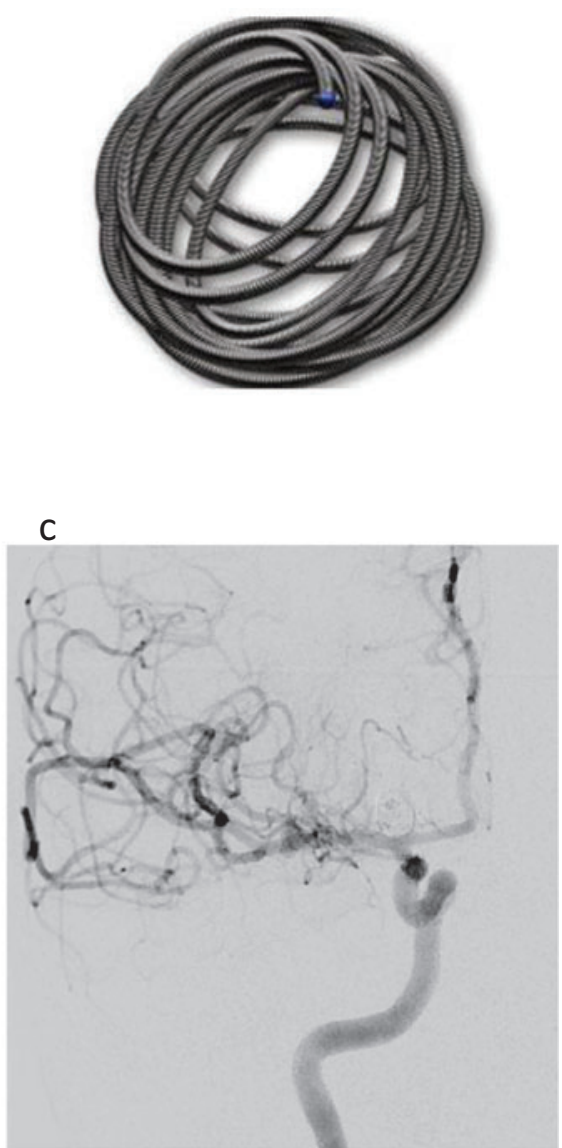

Figure 1 Frontal right internal carotid arterial projections (A: pre-embolisation; B: following placement of a microcatheter (arrow) into the lumen and a balloon catheter (thick arrow) across the neck to prevent coil prolapse: C: postembolisation) in 37-year-old woman with a $6 \mathrm{~mm}$ irregular unruptured right terminal internal carotid artery aneurysm. She is a smoker. D: diagram of a platinum coil. 

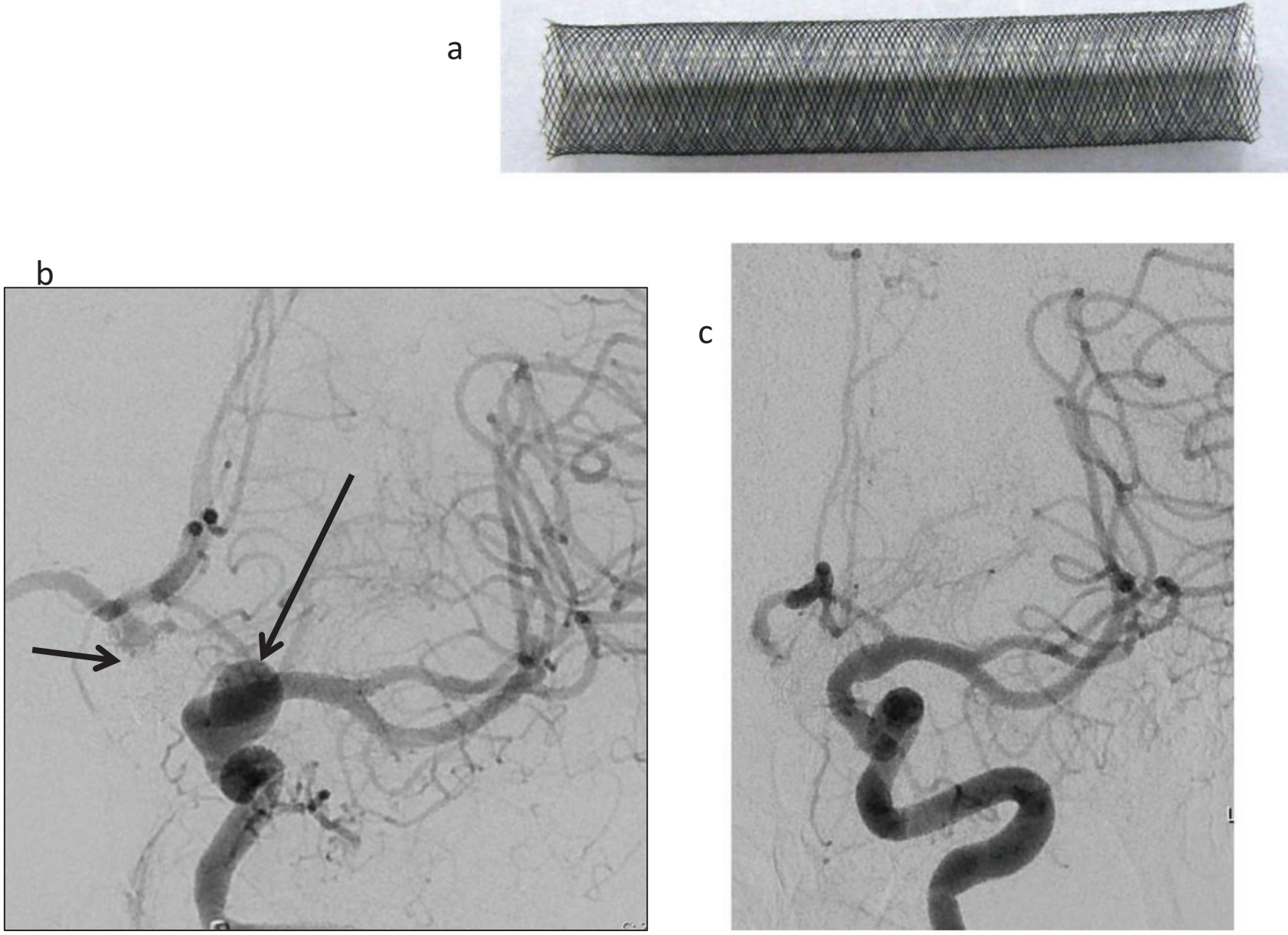

Figure 2 Sixty-one-year-old woman with a previous coiled anterior communicating artery aneurysm (short arrow). She underwent elective endovascular treatment of an incidental $7-8 \mathrm{~mm}$ left para-ophthalmic aneurysm (B, long arrow) with a relatively wide neck, using a Pipeline Shield Flow diverting device (A). Frontal angiographic projections of a left internal carotid angiogram pre-embolisation (B) and at 6-month follow-up (C) show complete aneurysmal occlusion.

events occurred in $7.6 \%$ and procedural aneurysmal perforation in $2.6 \%$ of cases.

A recent meta-analysis and systematic review of both neurosurgical and endovascular interventions ${ }^{25}$ examined 114 studies comprising 106433 patients and 108263 UIAs. There were 74 endovascular therapy studies with a complication rate of nearly 5\% (95\% CI $4 \%$ to $6 \%)$ and case fatality risk of $0.3 \%$ ( $95 \%$ CI $0.2 \%$ to $0.4 \%)$. Factors associated with complications were female sex, diabetes mellitus, hyperlipidaemia, cardiac comorbidities, aneurysm neck $>4 \mathrm{~mm}$ or dome neck ratio $>1.5$, posterior circulation location and stent-assisted coil embolisation. Aneurysm size $>10 \mathrm{~mm}$ also appears to be a risk factor for procedural complications.

\section{NEUROSURGICAL TREATMENT}

Neurosurgical interventions typically involve standard microsurgical clipping or clip reconstruction. It is rare to embark on more complex procedures, such as a bypass, for an unruptured aneurysm.

A systematic review and meta-analysis of 54 neurosurgical studies ${ }^{25}$ reported a complication rate of $8.34 \%$ (95\% CI $6.25 \%$ to $11 \%$ ) and a case fatality rate of $0.1 \%$
(95\% CI $0 \%$ to $0.2 \%)$. Factors associated with complications included age $>50$ years, female sex, coagulopathy, anticoagulation, smoking, hypertension, diabetes mellitus, congestive heart failure, posterior circulation location, aneurysm calcification and aneurysm size $>12 \mathrm{~mm}$.

Another meta-analysis of 60 surgical studies (nine of high quality) including 9845 patients and 10845 UIAs $^{26}$ reported unfavourable outcomes including death in $6.7 \%$ of cases (95\% CI $4.9 \%$ to $9.0 \%$ ).

There is no reported randomised controlled trial comparing endovascular treatment with neurosurgical treatment of UIAs. The International Subarachnoid Haemorrhage Trial reported better outcomes from coiling compared with clipping of ruptured aneurysms. However, this finding may not be applicable to UIAs. The Canadian Unruptured Endovascular versus Surgery trial, a pragmatic trial comparing endovascular therapy and surgery, is ongoing. ${ }^{27}$ An initial analysis has found no difference between morbidity and mortality after 1 year. New neurological deficits (OR: 3.12 $(1.05-10.56, \mathrm{p}=0.031))$ and hospital stay beyond 5 days (OR: $8.85(3.22-28.59, \mathrm{p}=0.0001))$ were significantly more frequent after clipping. 
b
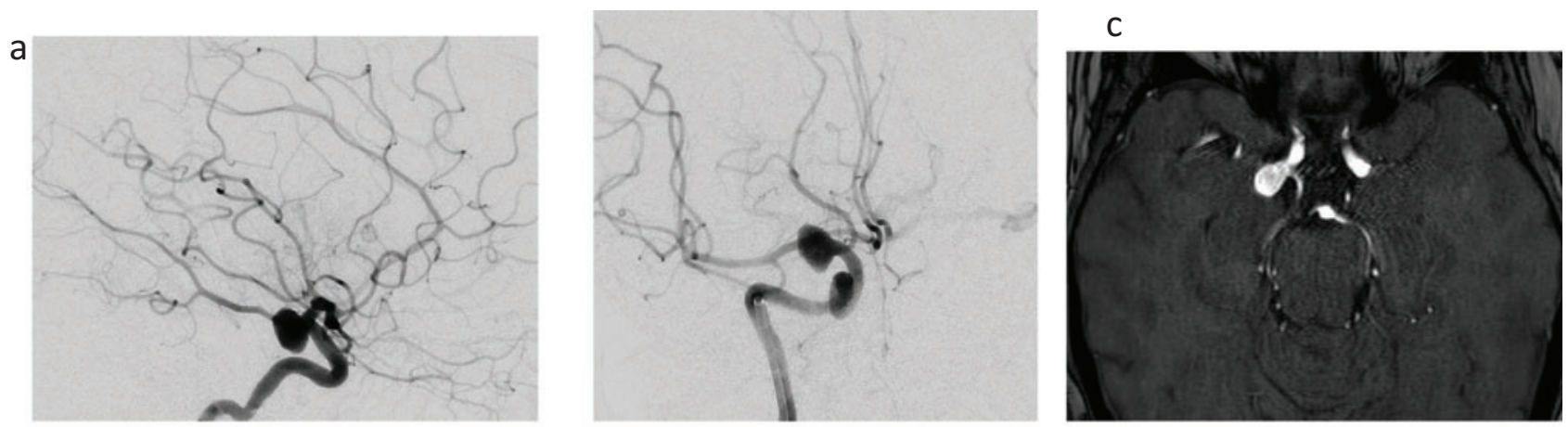

$\mathrm{d}$

e
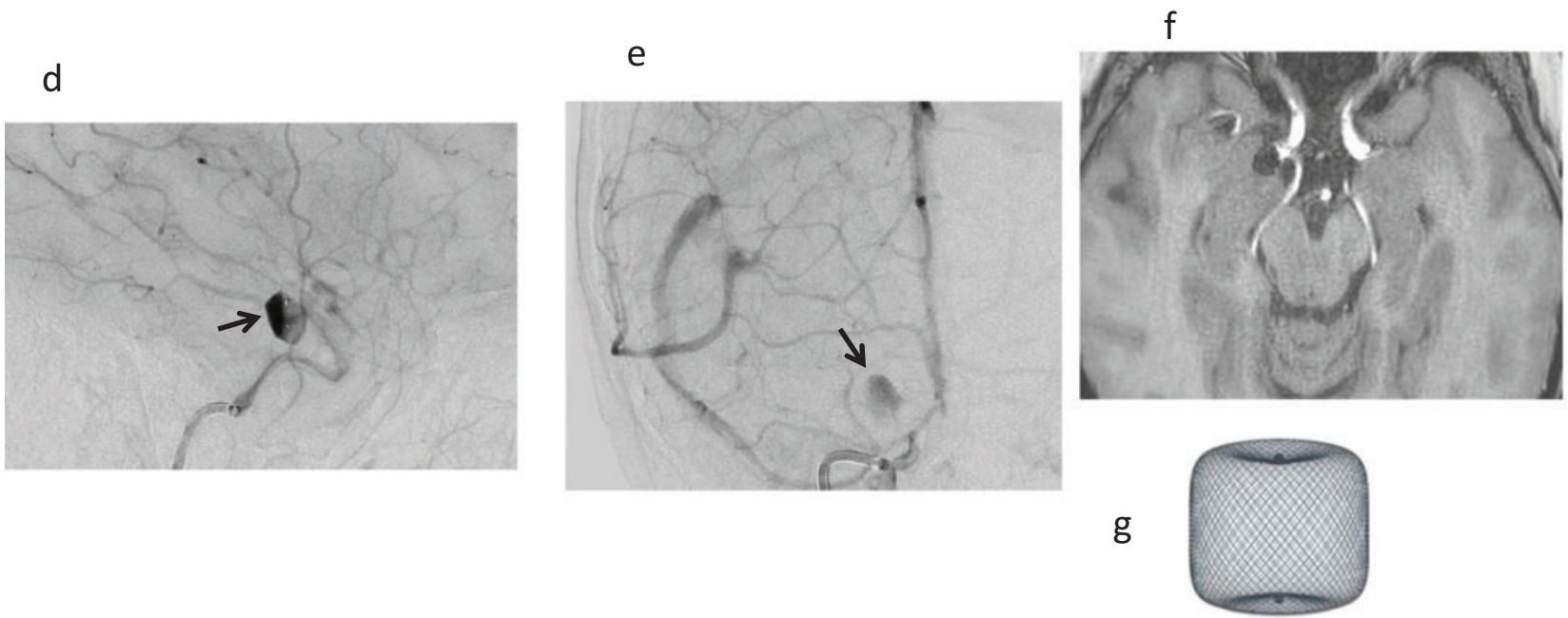

Figure 3 Forty-nine-year-old woman with hypertension and renal failure, on dialysis, awaiting renal transplant. Lateral (A,D) and frontal (B, E) projections of a right internal carotid artery angiogram show an unruptured $11 \mathrm{~mm}$ posterior communicating artery aneurysm, with a wide neck incorporating a fetal posterior cerebral artery: predeployment $(A, B)$ and postdeployment $(D, E)$ of a woven endobridge $($ WEB) device $(G)$. Note the intrasaccular flow disruption and stasis within the aneurysm immediately after deployment of the WEB (arrows). Six-month follow-up was with MR angiogram in view of her renal failure. 3D time-of-flight unmapped MR spectroscopy images prior (C) and 6 months later (F) confirm satisfactory aneurysmal occlusion (thanks to Dr Robert Crossley for permission to use images).

Since neurosurgery carries more risk with aneurysms that are large, in the posterior circulation and in older patients, the treatment of choice is generally considered to be endovascular treatment, particularly if simple or assisted coiling is possible.

\section{CONSERVATIVE MANAGEMENT}

The principal conservative measures are smoking cessation and controlling blood pressure. The American Heart Association guidelines recommend maintaining a systolic blood pressure of $<140 \mathrm{~mm} \mathrm{Hg}{ }^{28}$

Aspirin may decrease aneurysm wall inflammation and decrease formation, growth and rupture risk. Studies have shown that patients taking aspirin for other indications had a lower risk of UIA rupture. ${ }^{29}$ The PROTECT$\mathrm{U}$ study is investigating whether daily aspirin intake and controlled systolic blood pressure $(<120 \mathrm{~mm} \mathrm{Hg})$ reduce the risk of UIA rupture or growth.

Serial non-invasive angiographic imaging (MR angiography or CT angiography) is generally recommended to assess the potential growth of a conservatively managed UIA. American Heart Association guidelines ${ }^{28}$ suggest follow-up at 6-12 months and then imaging either yearly or biennially (Class IIB level of evidence). However, there is a case for using ELAPSS to assess the growth risk and tailoring the scan interval accordingly.

The growth rates of aneurysms $<4 \mathrm{~mm}$ are extremely low and so follow-up imaging for aneurysms $\leq 3 \mathrm{~mm}$ is disputed. Data from a comparative effectiveness study on management of UIA $\leq 3 \mathrm{~mm}$ showed that no invasive treatment and no follow-up gave the highest societal health benefit. ${ }^{30}$

MR angiography is the best modality for follow-up as it involves neither ionising radiation nor intravenous contrast. Its sensitivity for detecting aneurysms $\geq 3 \mathrm{~mm}$ is $87-100 \%$ and sp withecificity is $86-100 \%$. $3 \mathrm{~T}$ MR is better than $1.5 \mathrm{~T}$ MR, reflecting the higher spatial resolution $(0.6 \mathrm{~mm})$ and signal-to-noise ratio. CT angiography has similar sensitivities $(92.8-100 \%)$ and specificities $(83-100 \%)^{31}$ for aneurysms $\geq 3 \mathrm{~mm}$. 
Recent observational studies have suggested that intracranial aneurysm wall enhancement using highresolution vessel wall MR is a possible biomarker for aneurysm instability and rupture and that absence of wall enhancement strongly indicates aneurysm stability. ${ }^{32}$ Wall enhancement is more common in unstable and large aneurysms ${ }^{33}$ but may also occur in a low percentage of small unruptured and stable aneurysms. ${ }^{34}$ Commonly reported histopathological correlates for enhancement include the presence of atherosclerosis, active macrophages, neovascularisation and decreased elastin. Of interest, UIAs with a PHASES score $>3$ are more likely to have wall enhancement. However, high-resolution vessel wall MR has not been validated in clinical studies and should not be used for clinical decision-making. The study sequences and protocols used vary considerably and there is no consensus on the definition of wall enhancement whether circumferential, partial, focal or strong. Flow artefacts, contrast extravasation and stagnant flow can mimic aneurysm wall enhancement.

\section{DECISION-MAKING}

Each time a UIA is diagnosed, a neurovascular multidisciplinary team must reach a consensus on the risk of aneurysmal rupture, the risks of endovascular and surgical treatment, and the durability of treatment, taking into account the patient's age, comorbidities, lifestyle and personal wishes. The ultimate goal is to maximise a patient's quality-adjusted life years.

Some frameworks can help the decision-making process. The unruptured intracranial aneurysm treatment score $^{35}$ (UIATS, table 3) was derived from a multidisciplinary group of 69 neurovascular specialists using a Delphi consensus to develop a comprehensive scoring model for management recommendations for UIAs. The model takes into account patient-related, aneurysm-related and treatment-related risk factors (figure 4) in the decisionmaking process. A difference of $\geq 3$ points provides a recommendation for individual management. If the difference is $\leq 2$, a definitive recommendation cannot be made and either management approach could be supported. The model is complex and not yet validated.

A simpler treatment score may be more accurate than the UIATS judged by their respective receiver operating characteristic curves. ${ }^{36}$ The score is based upon a unique series of 142 patients diagnosed between 1956 and 1978 whose UIAs were managed, as a matter of policy, conservatively with a median follow-up of 21 years, thus reducing selection bias. There were 34 aneurysmal ruptures during 3064 person years of follow-up in patients largely of working age.

The treatment score comprises four variables: age $<40$ years ( 2 points), current smoking ( 2 points), UIA size $7 \mathrm{~mm}$ and larger ( 3 points) and location (anterior

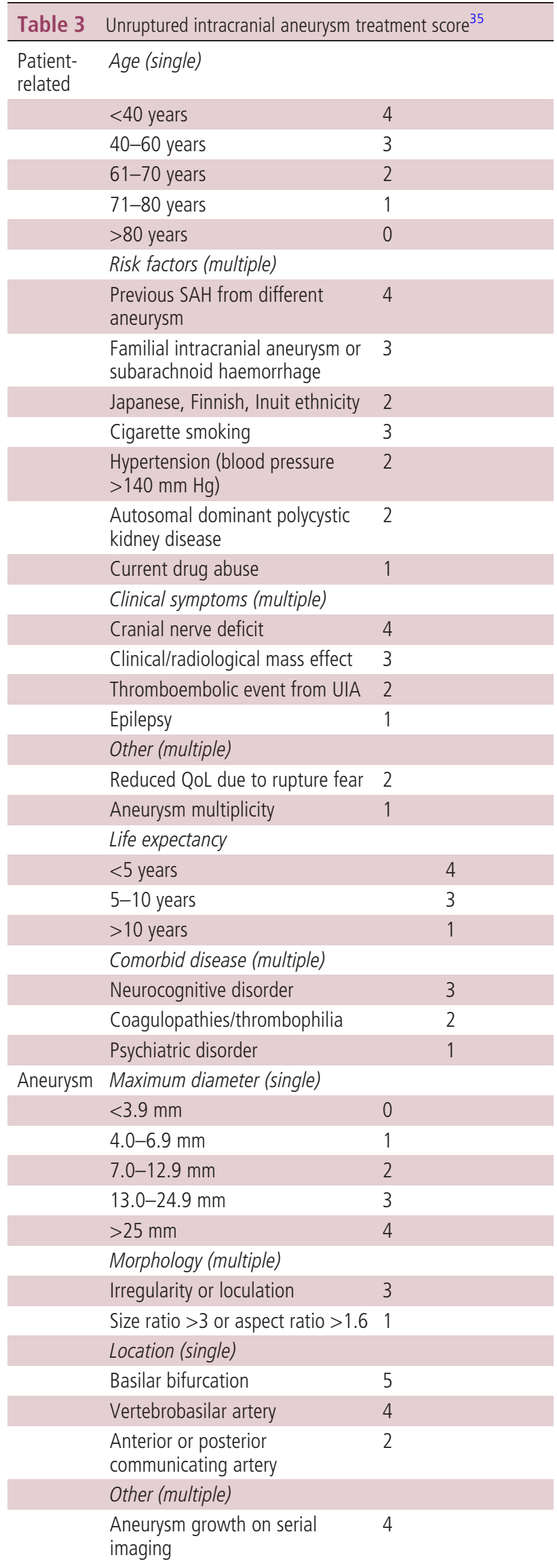




\begin{tabular}{|c|c|c|c|}
\hline & $\begin{array}{l}\text { Aneurysm de novo formation on } \\
\text { serial imaging }\end{array}$ & 3 & \\
\hline & $\begin{array}{l}\text { Contralateral steno-occlusive } \\
\text { disease }\end{array}$ & 1 & \\
\hline \multirow[t]{17}{*}{ Treatment } & Age-related risk & & \\
\hline & $<40$ years & & 0 \\
\hline & $41-60$ years & & 1 \\
\hline & $61-70$ years & & 3 \\
\hline & $71-80$ years & & 4 \\
\hline & $>80$ years & & 5 \\
\hline & Aneurysm size-related risk & & \\
\hline & $<6.0 \mathrm{~mm}$ & & 0 \\
\hline & $6.0-10.0 \mathrm{~mm}$ & & 1 \\
\hline & $10.1-20.0 \mathrm{~mm}$ & & 3 \\
\hline & $>20 \mathrm{~mm}$ & & 5 \\
\hline & Aneurysm complexity-related risk & & \\
\hline & High & & 3 \\
\hline & Low & & 0 \\
\hline & Intervention-related risk onstant & & 5 \\
\hline & Total score & & \\
\hline & Score favours UIA & Repair & Conservative \\
\hline
\end{tabular}

communicating 5 points, internal carotid bifurcation 4 points, posterior communicating 2 points). Scores of 5-12 were associated with a high cumulative risk of rupture $(16-60 \%$ at 10 years; $49-80 \%$ at 30 years), favouring UIA occlusion. A score of 1-4 was associated with a much lower cumulative risk of rupture $(3 \%$ at 10 years, $18 \%$ at 30 years), favouring conservative management unless there were additional indications for treatment. A score of 0 was associated with no ruptures in 513 patient-years of follow-up and such UIAs should not be treated. Thus, a 45 -year-old non- smoker with a $6 \mathrm{~mm}$ internal carotid aneurysm should receive conservative management in the absence of other risk factors.

The treatment score was devised predominantly to assist in the management of UIAs in working-age patients and should be applied with common sense. It would be inappropriate to recommend treatment of all anterior communicating artery aneurysms regardless of size and patient age or for any $\geq 7 \mathrm{~mm}$ aneurysm in an 80 -year-old smoker.

\section{SCREENING}

The rationale for screening for UIAs is to prevent aneurysmal subarachnoid haemorrhage, but the benefits of screening are far from clear cut. Most UIAs detected by screening are small and the optimal management for many of those detected may be conservative. The risks of treating a technically difficult small UIA may heavily outweigh the natural history risk. Yet following diagnosis, the patient will be exposed to the psychological stress of harbouring a UIA. We cannot say with certainty that single small aneurysms detected in all at-risk groups have higher rupture rates than sporadic UIAs. Since aneurysm growth is non-linear, the timing of follow-up scans in patients managed conservatively is problematic. In some jurisdictions, a UIA may have implications for insurance. For all these reasons, patients should be carefully counselled before undergoing screening.

Patient groups at risk of aneurysm formation include those with a family history of intracranial aneurysms or aneurysmal subarachnoid haemorrhage, autosomal dominant polycystic kidney disease, Ehlers-Danlos syndrome type 4, Marfan's syndrome, coarctation of the aorta, bicuspid aortic valve, hereditary haemorrhagic telangiectasia, pseudoxanthoma elasticum, neurofibromatosis type 1, alpha-1 antitrypsin deficiency, fibromuscular dysplasia, phaeochromocytoma, tuberous sclerosis and Noonan's

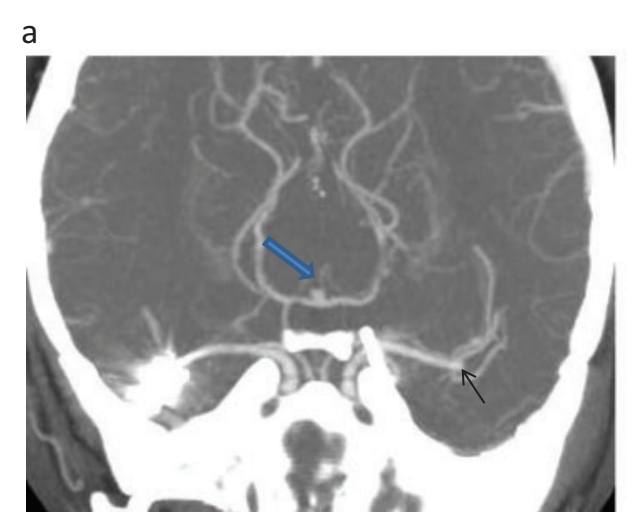

$b$

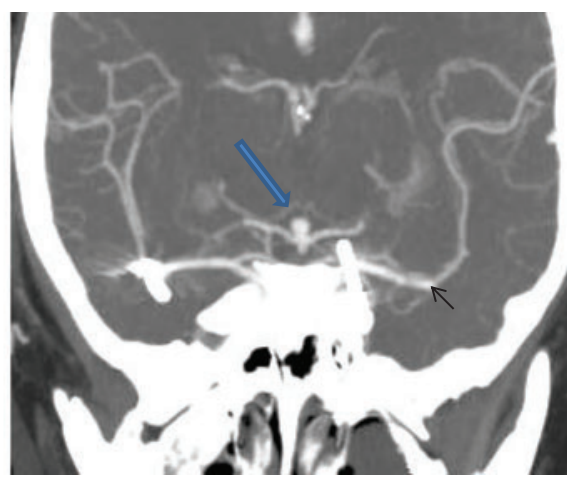

Figure 4 Coronal CT angiographic reconstructions of a 43-year-old non-smoking, normotensive woman (who had ruptured left ophthalmic and right middle cerebral aneurysms clipped at the ages of 6 and 30 respectively) show an enlarging basilar aneurysm (A) $<3 \mathrm{~mm}$ in 2015 (B) to $4.5 \mathrm{~mm}$ in 2020 (open blue arrow) and a small stable left middle cerebral artery aneurysm (solid arrow). Neither of these aneurysms was present in 2007. The PHASES-based 5-year rupture risk has increased from 1.3\% in 2015 to 5\% in 2020. The 2020 ELAPSS score predicts a 3-year growth risk of $8 \%$ and a five year risk of 13\%. The 2020 UIATS score favours aneurysm repair. This case illustrates the importance of multi-factorial decision making. Although the patient's basilar aneurysm remains small $(<5 \mathrm{~mm})$ and she has no cardiovascular risk factors; the site and growth of the aneurysm, her previous subarachnoid haemorrhages and the presence of multiple aneurysms favour treatment. 
syndrome. In these at-risk groups, screening is generally recommended for familial aneurysms, autosomal dominant polycystic kidney disease and aortic coarctation. ${ }^{37}$

The prevalence of an UIA in patients with one affected family member is around 4\%, marginally higher than the general population, and screening is not recommended. Current guidelines recommend screening patients with two or more first-degree relatives with a UIA or aneurysmal subarachnoid haemorrhage where the prevalence of incidental UIAs is $8-10 \%$, in those 30 years and older. The risks are higher in siblings, in females, cigarette smokers and hypertensives. Non-invasive angiography should begin at 20 years of age and repeated every 5 years, since aneurysms may develop even after two negative screens. ${ }^{38}$

Familial aneurysms rupture at a younger age; they may anticipate a generation and may rupture at a smaller size than sporadic aneurysms. The increased rupture risk for size is around three times. ${ }^{39}$ The prevalence of UIAs increases substantially in those who smoke and have hypertension; $19 \%$ of family members with these risk factors were found to have at least one UIA. The rate of haemorrhage in familial UIAs with these risk factors may be 17 times higher, even in aneurysms as small as $3 \mathrm{~mm} .{ }^{40}$

Screening is also recommended for patients with autosomal dominant polycystic kidney disease where the prevalence is around $10 \%$, rising to $20 \%$ in those with a family history of UIA or aneurysmal subarachnoid haemorrhage. Ten per cent of patients with aortic coarctation have an UIA and screening is also recommended. It is unknown whether hypertension is the underlying cause or another pathogenic mechanism.

Neurologists are often asked by patients or their general practitioners about the indications for screening for UIAs and may question whether it is appropriate for them to initiate the screening themselves. Many neurovascular multidisciplinary teams have published guidelines on screening and whether they prefer to review all patients before proceeding with screening. Screening is usually done in patients with two or more affected first-degree relatives and those associated with autosomal dominant polycystic kidney disease. However, the extent of screening in any family lineage, the timing of further screening, the consequences of identifying an aneurysm and particularly small, multiple and complex aneurysms may all raise difficult issues. Thus, unless a neurologist has a special interest in neurovascular disorders, referral to neurovascular multidisciplinary team would seem appropriate.

\section{CONCLUSION}

The management of individuals with incidental unruptured intracranial aneurysms is both complex and nuanced and requires pragmatism and common sense, taking into account mainly age, comorbidities, life expectancy, aneurysm size and location, and the risks and difficulties anticipated with the intervention.

\section{Key points}

- Unruptured intracranial aneurysms (UIAs) affect 3\% of the adult population in most Western countries and most do not rupture.

- The principal risks for rupture are size, location, smoking, hypertension and interval growth.

- The risks associated with endovascular or neurosurgical treatment often exceed the natural history risk, especially with small UIAs.

- If the neurovascular multidisciplinary team does not consider UIA treatment to be beneficial, or if the patient declines it after a detailed discussion of risk and benefit, then conservative management should include treatment of vascular risk factors and noninvasive angiographic follow-up in selected cases.

- In general, UIAs with a diameter $>5 \mathrm{~mm}$ in younger patients should be considered for treatment especially if irregular, enlarging and with a family history.

- There is usually no benefit to invasive treatment or follow-up in elderly and frail patients with small aneurysms - they have better things to do.

Funding The authors have not declared a specific grant for this research from any funding agency in the public, commercial or not-for-profit sectors.

Competing interests None declared.

Patient consent for publication Not required.

Provenance and peer review Commissioned. Externally peer reviewed by Teddy Wu, Christchurch, New Zealand.

\section{REFERENCES}

1 Vlak MH, Algra A, Brandenburg R, et al. Prevalence of unruptured intracranial aneurysms, with emphasis on sex, age, comorbidity, country and time period: A systematic review and meta-analysis. Lancet Neurol 2011;10:626-36.

2 Etminan N, Chang H-C, Algra A. Worldwide incidence of aneurysmal subarachnoid haemorrhage according to region, time period, blood pressure and smoking prevalence in the population. JAMA Neurol 2019;76:588-97.

3 Chalouhi N, Ali MS, Jabbour PM, et al. Biology of intracranial aneurysms: role of inflammation. J Cereb Blood Flow Metab 2012;32:1659-1676.

4 Chalouhi N, Hob B, Hasan D. Review of aneurysm formation, growth and rupture. Stroke 2013;44:3613-22.

5 Greving JP, Wermer MJ, Brown RD, et al. Development of the PHASES score for prediction of risk of rupture of intracranial aneurysms: a pooled analysis of six prospective cohort studies. Lancet Neurol 2014;13:59-66.

6 Wiebers DO, Whisnant JP, Huston J 3rd, et al. Unruptured intracranial aneurysms: natural history, clinical outcome and risks of surgical and endovascular treatment. Lancet 2003;362:103-10.

7 Bijlenga P, Gondar R, Schilling S, et al. PHASES score for the management of intracranial aneurysm. Stroke 2017;48:2105-12.

8 Choi HH, Cho YD, Jeon JP, et al. Growth of untreated unruptured small-sized aneurysms $(<7 \mathrm{~mm})$ : incidence and related factors. Clin Neuroradiol 2018;28:183-9. 
9 Murayama Y, Takao H, Ishibashi T, et al. Risk analysis of unruptured intracranial aneurysms. Stroke 2016;47:365-71.

10 Backes D, Rinkel GJE, Grieving JP, et al. ELAPSS score for prediction of risk of growth of unruptured intracranial aneurysms. Neurology 2017;88:1600-1606.

11 Brinjikji W, Zhu YQ, Lanzino G, et al. Risk factors for growth of intracranial aneurysms: a systematic review and meta-analysis. Am J Neuroradiology 2016;37:615-20.

12 Mehan WA, Romero JM, Hirsch JA, et al. Unruptured intracranial aneurysms conservatively followed with serial CT angiography: could morphology and growth predict rupture? J Neurointerventional Surg 2014;6:761-6.

13 Etminan N, Rinkel GJ. Unruptured intracranial aneurysms: development, rupture and preventative management. Nat Rev Neurol 2017;13:126-41.

14 Juvela S, Poussa K, Lehto H, et al. Natural history of unruptured intracranial aneurysms. Stroke 2013;44:2414-21.

15 Brinjikji W, Pereira VM, Khumtong R, et al. PHASES and ELAPPS scores are associated with aneurysm growth: a study of 431 unruptured aneurysms. World Neurosurg 2018;114:e425-432.

16 Giordan E, Sorenson TJ, Brinjikji W, et al. Risk factors for growth of conservatively managed unruptured intracranial aneurysms. Acta Neurochir (Wien) 2018;160:2419-23.

17 Backes D, Vergouwen MDI, Groenestege ATT, et al. PHASES score for prediction of intracranial aneurysm growth. Stroke 2015;46:1221-6.

18 Ujiie $\mathrm{H}$, Tamanio Y, Sasaki K, et al. Is the aspect ratio a reliable index for predicting the rupture of a saccular aneurysm? Neurosurgery 2001;48:493-5.

19 Hassan T, Timofeev EV, Saito T, et al. A.proposed vessel geometry-based categorization of saccular intracranial aneurysms: computational flow dynamics analysis of the risk factors for lesion rupture. J Neurosurgery 2005;103:662-80.

20 Raymond J, Roy D. Unruptured intracranial aneurysms and the trial on endovascular management (TEAM): the principles behind the protocol. J Vasc Interventional Neurol 2008;1:22-6.

21 Pierot L, Spelle L, Vitry F. for the ATENA investigators. Immediate clinical outcome of patients harbouring unruptured intracranial aneurysms treated by endovascular approach: results of the ATENA study. Stroke 2008;39:2497-504.

22 Brinjikji W, Murad MH, Lanzino G, et al. Endovascular treatment of intracranial aneurysms with flow divertors: a metaanalysis. Stroke 2013;44:442-7.

23 Pierot L, Gawlitza M, Soize S. Unruptured intracranial aneurysms: management strategy and current endovascular treatment options. Expert Rev Neurother 2017;10:977-86.

24 Naggara ON, White PM, Guilbert F, et al. Endovascular treatment of intracranial unruptured aneurysms: systematic review and meta-analysis of the literature on safety and efficacy. Radiology 2010;256:887-97.
25 Algra A, Lindgren A, Vergouwen M, et al. Procedural clinical complications, case fatality risks and risk factors in endovascular and neurosurgical treatment of unruptured intracranial aneurysms. JAMA Neurol 2019;76:282-91.

26 Kotowski M, Naggara O, Darsault TE, et al. Safety and occlusion rates of surgical treatment of unruptured aneurysms: a systematic review and meta-analysis of the literature from 1990-2011. J Neurol Neurosurgery Psychiatry 2013;84:42-8.

27 Darsault TE, Findlay JM, Magro E, et al. Surgical clipping or endovascular coiling for unruptured intracranial aneurysms: a pragmatic randomised trial. J Neurol Neurosurgery Psychiatry 2017;88:663-8.

28 Thompson BG, Brown RD, Amin-Hanjani S, et al. Guidelines for the management of patients with unruptured intracranial aneurysms. Stroke 2015;4:2368-400.

29 Hudson JS, Marincovich AJ, Roa JA, et al. Aspirin and intracranial aneurysms. Stroke 2019;50:2591-6.

30 Malhotra A, Wu X, Forman HP, et al. Management of tiny unruptured intracranial aneurysms: a comparative effectiveness analysis. JAMA Neurol 2018;75:27-34.

31 Rodriguez Regent C, Edjlali-Goujon M, Boulouis G, et al. Noninvasive diagnosis of intracranial aneurysms. Diagn Interv Imaging 2014;95:1163-74.

32 Texakalidis P, Hilditch CA, Lehman V, et al. Vessel wall imaging of intracranial aneurysms: systematic review and meta-analysis World. Neurosurgery 2018;117:453-8.

34 Vergouwen MDI, Backes D, van der Schaaf IC, et al. Gadolinium enhancement of the aneurysm wall in unruptured intracranial aneurysms is associated with an increased risk of aneurysm instability: a follow up study. Am J Neuroradiology 2019;40:1112-16.

33 Samaniego EA, Roa JA, Hasan D. Vessel wall imaging in intracranial aneurysms. J Neurointerv Surg 2019;11:1105-12.

35 Etminan N, Brown RD, Beseoglu K, et al. The unruptured intracranial aneurysm treatment score. Neurology $2015 ; 85: 881-9$.

36 Juvela S. Treatment scoring of unruptured intracranial aneurysms. Stroke 2019;50.

37 Rinkel GJ. Intracranial aneurysm screening: indications and advice for practice. Lancet Neurol 2005;4:122-8.

38 Bohr AS, Rinkel GJ, van Norden J, et al. Long-term screening for intracranial aneurysms in individuals with a family history of aneurysmal subarachnoid haemorrhage: a cohort study. Lancet Neurol 2014;13:385-92.

39 Mensing LA, Greving JP, Verhoeff TA, et al. Comparison of rupture risk of intracranial aneurysms between familial and sporadic patients. Stroke 2019;50:1380-3.

40 Broderick JP, Brown RD, Sauerbeck L, et al. Greater rupture risk for familial as compared to sporadic unruptured intracranial aneurysms. Stroke 2009;40:1952-1957. 